\section{P42 THE RELATIONSHIP OF HOME ACTIVITY LEVELS TO PSYCHOLOGICAL CO-MORBIDITY IN COPD}

doi:10.1136/thoraxjnl-2011-201054c.42

${ }^{1} \mathrm{~T}$ Stuart, ${ }^{2} \mathrm{C}$ Rogers, ${ }^{2} \mathrm{G}$ Balanos, ${ }^{2} \mathrm{~A} \mathrm{M}$ Wood. ${ }^{1}$ Heart of England NHS Foundation Trust, Birmingham, UK; ${ }^{2}$ University of Birmingham, Birmingham, UK

Background Physical activity levels are often reduced in patients with COPD, but are partially amenable to intervention, predominantly by rehabilitation. Depression and anxiety are also commonly observed co-morbidities, which relate to outcomes such as mortality and admission rates. ${ }^{1}$ A recent meta-analysis of exercise as a treatment for depression suggested that it may improve depressive symptoms, ${ }^{2}$ hence it is recommended by NICE guidance for mild depression. We hypothesised that less active COPD patients would also have higher rates of psychological co-morbidity.

Methods 59 consecutive COPD admissions or attendees to COPD clinic were recruited between January and March 2010, screened for anxiety and depression, and questioned regarding activity levels using the Modified Baecke Questionnaire (MBQ). Those who were already on treatment for anxiety or depression were excluded from further analysis $(n=4)$. Home activity monitoring using the Actigraph was conducted in 20 patients and 6MWT distances recorded.

Results $38.2 \%$ of patients were anxious and $36.4 \%$ depressed, according to HADS. Both related strongly to exacerbation and admission rates over the preceding 12 months $(p<0.01)$, and to $\mathrm{QOL}$ as measured by CAT $(p<0.01)$. Perceived activity as measured by $\mathrm{MBO}$ was lower in depressed patients $(\mathrm{p}=0.02)$; this remained significant after regression analysis, adjusting for exacerbations and $\mathrm{FEV}_{1}(\mathrm{p}=0.03)$. However, actual activity from the Actigraph showed no relationship to either anxiety or depression (both $p>0.38$ ).

Conclusions Depressed patients perceive their symptoms to be more severe, and therefore their activity levels lower, than non-depressed patients. However, this did not translate into a difference in actual activity levels. Whether exercise programmes can impact on psychological co-morbidity in COPD requires further research.

\section{REFERENCES}

1. Maurer J, et al. Chest. 2008;134:43S-56S

2. Mead GE, et al. Cochrane Database Syst Rev 2009:CD004366.

\section{P43 ASSESSING THE VALIDITY OF A HOME ACTIVITY MONITOR IN PATIENTS WITH COPD}

doi:10.1136/thoraxjn|-2011-201054c.43

C Rogers, G Balanos, A M Wood. University of Birmingham, Birmingham, UK

Background Self-reported physical activity equivalent to at least $2 \mathrm{~h}$ a week is associated with reduction in the risk of hospital admission and death in COPD. ${ }^{1}$ However, whether self reported activity is accurate in COPD is uncertain. The gold standard objective measures of physical activity in daily life are direct observation and assessment of energy expenditure by calorimetry or the doubly labelled water method. ${ }^{2}$ We sought to test the validity of a home activity monitor (Actigraph) - for measurement of physical activity levels by comparing to calorimetry, the 6MWT and an activity questionnaire.

Methods 20 patients with COPD and five healthy elderly subjects underwent home activity monitoring over a period of 5 days, a $6 \mathrm{MWT}$ and were questioned twice regarding activity levels using the modified Baecke questionnaire (MBQ). ${ }^{3}$ Time spent non-sedentary according to the Actigraph was compared to perceived activity from the $\mathrm{MBO}$ and $6 \mathrm{MWT}$. A sub-group also underwent concurrent measurement of energy expenditure by indirect calorimetry and the Actigraph during a walk at steady state on a treadmill.
Results Actigraph output is summarised in Abstract P43 table 1 $M B Q$ score did not relate to actual time active in patients $(p=0.54)$, and was less reproducible on retest than in controls. Furthermore when patients were ranked according to activity level by $\mathrm{MBO}$ and Actigraph, it was clear that patients both under and over-estimated their activity level. Actigraph activity levels related well to 6MWT $(p=0.04)$. Accuracy of the Actigraph at measuring energy expenditure, compared to the gold standard of calorimetry, varied with walking speed, such that it was less accurate in those walking slowly.

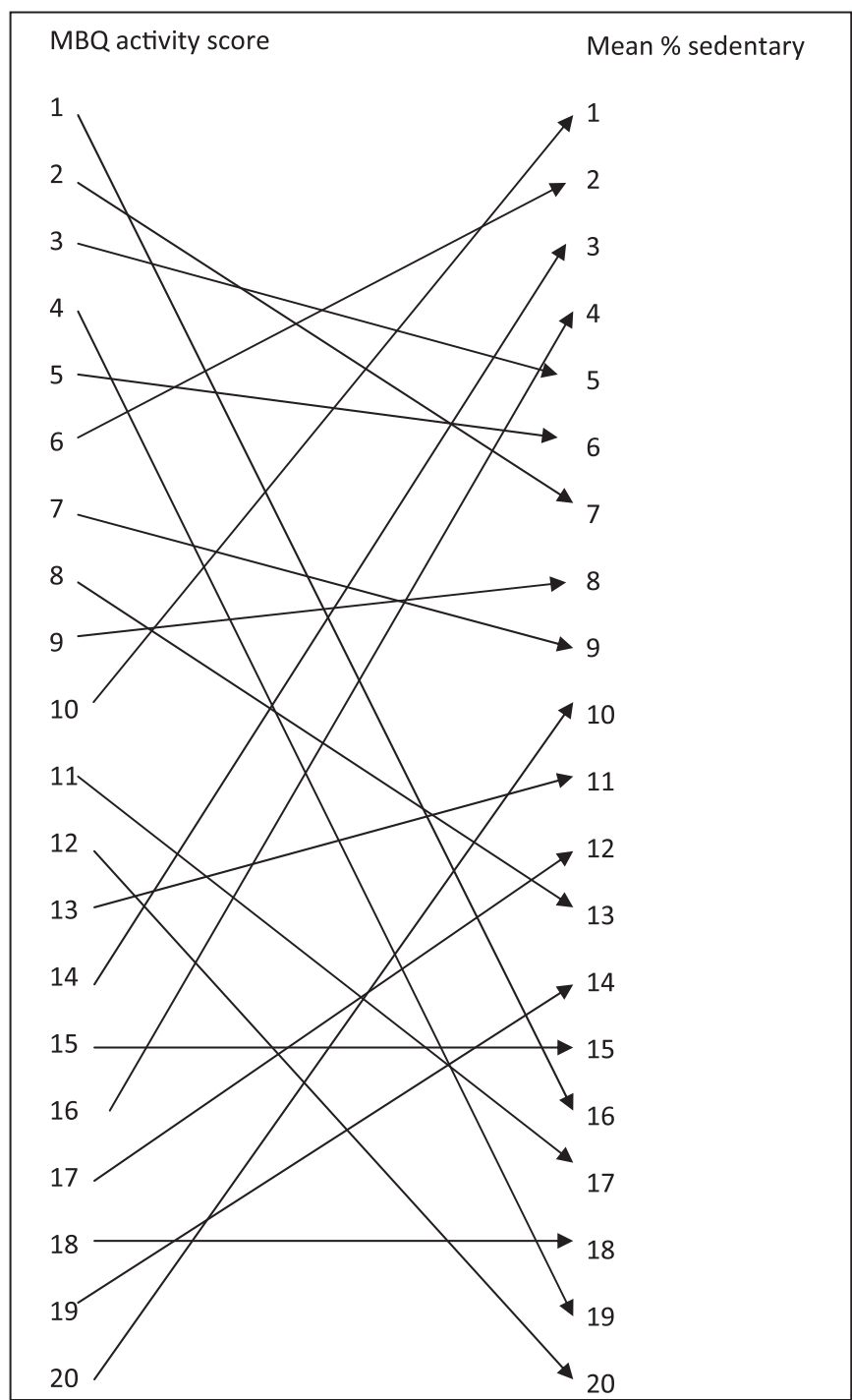

Abstract P43 Table 1 Relationship between MBO score and the percentage time spent sedentary. MBO score was ranked from highest to lowest (1-20). The mean \% time sedentary was ranked lowest to highest for the twenty patients. The arrows show how MBO score rank related to mean \% time sedentary. For example, the patient with the highest score on MBO was ranked 16th in the \% time sedentary, suggesting that they actually did less activity than they perceived they did. The patient who spent the least time sedentary perceived they did less activity than they actually did as they were ranked 10th for MBO score. Nine patients (45\%) over-estimated their activity level and nine under-estimated their activity level. Two patients were ranked the same for the MBO as the Actigraph.

Conclusions Patients self reported activity levels are likely to be inaccurate. Home activity monitoring is accurate at measuring exercise capacity, but not at measuring energy expenditure, due to slow speed of movement. 